\title{
e-Migrinter
}

$8 \mid 2012$

Regards sur les migrations sud-asiatiques

Initiatives et stratégies spatiales. Le projet circulatoire face aux politiques publiques : l'exemple des Rroms et des Voyageurs du PoitouCharentes (France) et de la Wallonie (Belgique) Thèse soutenue le 01 juin 2011 à l'Université de Poitiers

\section{Céline Bergeon}

\section{OpenEdition}

Journals

Édition électronique

URL : https://journals.openedition.org/e-migrinter/619

DOI : 10.4000/e-migrinter.619

ISSN : 1961-9685

Éditeur

UMR 7301 - Migrinter

Édition imprimée

Date de publication : 4 avril 2012

Pagination : 112-115

ISSN : 1961-9685

Référence électronique

Céline Bergeon, «Initiatives et stratégies spatiales. Le projet circulatoire face aux politiques publiques l'exemple des Rroms et des Voyageurs du Poitou-Charentes (France) et de la Wallonie (Belgique) », eMigrinter [En ligne], 8 | 2012, mis en ligne le, consulté le 20 mai 2021. URL : http://

journals.openedition.org/e-migrinter/619; DOI : https://doi.org/10.4000/e-migrinter.619 
Initiatives et stratégies spatiales. Le projet circulatoire face aux politiques publiques : l'exemple des Rroms et des Voyageurs du Poitou-Charentes (France) et de la Wallonie (Belgique)

\section{Thèse soutenue par Céline Bergeon le 01 juin 2011 à l'Université de Poitiers}

D epuis 2000, la France dispose d'une législation spécifique concernant le stationnement de ceux que l'administration appelle communément les « Gens du voyage ». Cette loi impose aux communes de plus de 5000 habitants de réaliser un dispositif d'accueil ${ }^{1}$ pour ces familles dont le quotidien s'organise à partir d'une mobilité spatiale plus ou moins soutenue. Erigée en mode de vie, cette circulation fédère ainsi les activités économiques, religieuses ou encore familiales, et joue un rôle d'équilibre dans la vie des familles. En contrepartie de l'obligation des communes à accueillir les "Gens du voyage », ces derniers doivent impérativement stationner sur les aires d'accueil qui leur sont destinées. Il s'agit de lieux pouvant accueillir entre quinze et cinquante caravanes, et sur lesquels des actions de prise en charge (éducatives ou sociales par exemple) doivent être organisées par les communes accueillantes. En France, les «Gens du voyage» doivent donc stationner en des lieux identifiés par l'État sous peine d'un emprisonnement de six mois, d'une amende de 3750 euros, de la suspension du permis de conduire pour une durée maximum de trois ans et de la confiscation du véhicule tractant la caravane. Dix ans après la promulgation de la loi, à peine $50 \%$ des obligations et des besoins mentionnés dans les schémas départementaux ont été réalisés. $\mathrm{La}$ Wallonie, quant à elle, ne dispose pas de législation spécifique pour le stationnement des «Gens du voyage ». Elle traite l'arrêt de ces familles au cas par cas et souvent selon le

${ }^{1}$ Dispositif d'accueil qui doit être établi dans un schéma départemental d'accueil des Gens du voyage. bon-vouloir des bourgmestres. Cependant, depuis cinq ans environ, la Wallonie souhaite encadrer législativement la présence des «Gens du voyage » sur son territoire et ce, sur le modèle d'accueil français.

L'objectif principal de cette thèse est d'une part de comprendre les motivations réelles et surtout les significations de la circulation traditionnelle (que les «Gens du voyage » appellent «le voyage »), et d'autre part, de voir les répercussions de l'injonction législative dans le déroulement du voyage. Autrement dit, elle interroge les nouveaux comportements circulatoires développés en réponse à la loi de 2000 qui implante de la localisation arbitraire pour l'arrêt des familles. Cette recherche s'insère donc dans une démarche compréhensive de la mobilité des «Gens du voyage » et plus largement dans l'analyse des mécanismes qui soustendent les relations entre une population majoritaire et un groupe minoritaire vivant sur un même territoire. Ce travail révèle ainsi l'évolution du voyage et met ainsi en évidence les facettes avant et après la législation. En cela, les deux terrains de recherche choisis pour cette thèse doivent être considérés non pas comme deux lieux d'investigation distincts mais bien comme deux espaces en complémentarité. Si l'un encadre le stationnement, l'autre ne le gère pas encore, et c'est en ce sens que l'évolution du voyage et le rôle de la législation ont pu être saisis de façon pertinente.

Au-delà de la compréhension du voyage, et parce que les relations «majoritéminorité » ont une influence certaine sur l'épanouissement du quotidien, une attention 
spécifique est portée aux liens sociaux entre ces familles et ceux qu'elles appellent les «Gadjé » (ceux qui n'appartiennent pas à la communauté des «Gens du voyage »). Cette analyse est réalisée à partir de l'observation, de temps de rencontre, de réunions, de situations de conflits, mais également à partir d'un travail précis sur les représentations et les identifications. Ce dernier a notamment permis de révéler un réel «bricolage des identités » opéré par les "Gens du voyage » et de mettre au jour des processus d'identification et d'auto-dénomination complexes qui ont permis de remettre en cause des classifications pré-établies. En effet, si l'expression "Gens du voyage » est la plus couramment utilisée par l'administration, les acteurs ne l'emploient que très rarement et lui préfèrent celle de «Voyageurs » ou bien de «Rroms». D'ailleurs, l'élite rrom qui se constitue depuis quelques années revendique pleinement ce terme. Néanmoins, l'expression «Voyageurs» est la plus couramment utilisée dans le quotidien pour affirmer son appartenance et se différencier de la population majoritaire.
La nature des relations sociales et le poids des représentations ont également été mesurés à l'aide d'un travail sur plus de 200 articles de presse, recueillis entre 2005 et 2009, révélant ainsi des sujets plus ou moins récurrents.

L'accueil et le stationnement semblent des sujets propices à la diffusion dans le média presse. En effet, les articles traitant de ces thématiques sont plus que récurrents, et montrent ainsi une des préoccupations majeures des autorités compétentes et de la population locale. En ce sens, une réflexion spécifique sur l'hospitalité est engagée dans la thèse afin de cerner les mécanismes inhérents à l'accueil, tant d'un point de vue pratique (organisation de l'accueil) que d'un point de vue relationnel et social (cohabitation, etc.).

La démarche scientifique adoptée pour saisir l'évolution du voyage et le rôle de la législation dans cette transformation est qualitative. Entretiens, observations et immersion ont réellement permis de comprendre les mutations et les permanences dans les pratiques circulatoires. Les résultats révèlent quatre types de

Figure 1: Récurrence des thématiques sur les Voyageurs dans les articles de presse.

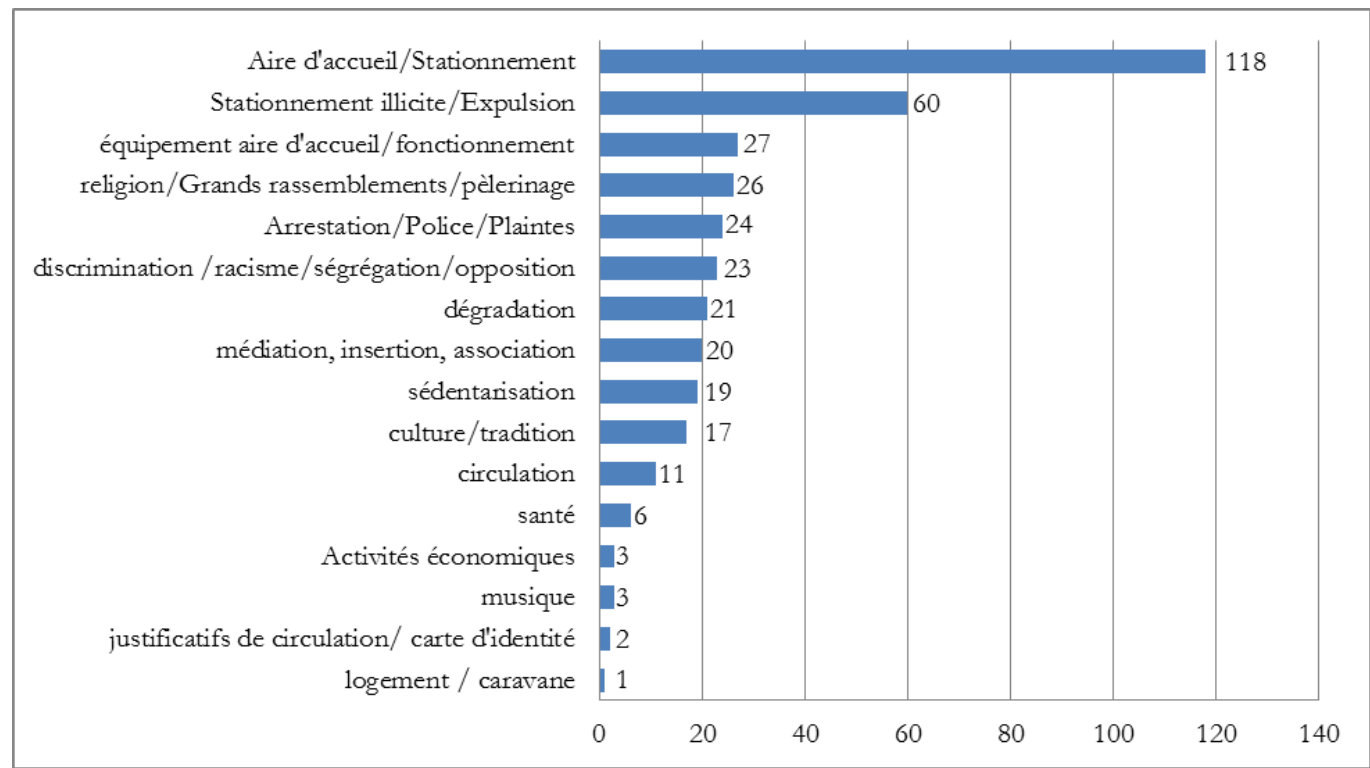

Source : Corpus d'articles de presse, 2005-2009. 
réponses à la législation : la soumission, la contestation, la sédentarisation et l'innovation, avec comme point commun, pour les Voyageurs, l'évidence que la loi de 2000 et la création des aires d'accueil ont désorganisé et déstructuré leur circulation.

Nombre de Voyageurs ont intégré les aires d'accueil tant dans leur parcours que dans leur quotidien. Ils se posent ainsi en situation de soumission par rapport à la loi. D'une fréquentation obligatoire, l'on est passé à une fréquentation «naturelle» au point que certains revendiquent pleinement ces espaces de stationnement comme leur propriété. D'autres les réclament lorsque la commune n'a pas respecté la loi, cette dernière devenant ainsi un outil de défense et de contestation.

Le deuxième type de comportement identifié est celui du refus et de la contestation de la loi, qui s'illustrent par du stationnement illicite volontaire. Les familles se posent ainsi en situation d'illégalité vis-àvis de leurs obligations de stationnement. La pratique illégale est ici utilisée pour signifier son désaccord avec le législateur et la société. Nombre de Voyageurs affirment alors que refuser de s'arrêter sur les aires d'accueil, c'est refuser l'homogénéisation du voyage, qui répond à l'évidence à des logiques différentes selon les familles.

Ces aires d'accueil et la complexification des conditions de la circulation ont eu des conséquences irrémédiables pour beaucoup de familles, au point que nombre d'entre elles se sont sédentarisées. Bien que le voyage ne soit pas totalement oublié (la présence de la caravane perdure malgré l'arrêt de la mobilité, le quotidien et les activités économiques restent le plus souvent identiques à celles pratiquées pendant la circulation), la sédentarisation est à l'initiative de familles qui ne réussissent plus à déployer leurs intentions circulatoires. Cette période de sédentarisation est néanmoins réversible et les Voyageurs évoquent le plus souvent une reprise future du voyage.

Enfin, de nombreuses familles font preuve d'innovation concernant leurs pratiques spatiales. La loi leur a alors permis de «revisiter le voyage». Le projet circulatoire a été mis en évidence et révèle le contournement, l'adaptation et l'autonomie dont font preuve ces familles pour perpétuer leur mode de vie. D'autres solutions à leur principale difficulté - le stationnement - sont envisagées comme celle par exemple de l'acquisition multisites. Les membres de la famille élargie achètent des «terrains familiaux » pouvant accueillir l'ensemble des caravanes de la famille. Ces espaces se situent sur l'itinéraire du parcours, facilitent de fait l'arrêt et réduisent les risques de la déstructuration du voyage.

L'analyse de la circulation, au regard de l'obstacle législatif, montre véritablement que le voyage ne relève pas de l'errance. Celui-ci est au contraire minutieusement établi, organisé et est dépendant de facteurs économiques, familiaux et religieux. L'attachement aux lieux du parcours, révélé par les entretiens, prouve, entre autres, le réel statut d'habitant des Voyageurs. Ils développent dans leur lieu de halte des habitudes de vie et de fréquentation qui procèdent véritablement au processus d'appropriation des lieux qu'ils investissent. En outre, la régularité de l'itinéraire, constatée très fréquemment pendant les entretiens, montre encore une fois que la circulation et la fréquentation des lieux sont des choix réfléchis et réactivés par la mémoire du voyage.

Cette analyse de la circulation laisse place, dans les derniers chapitres de cette thèse, à celle de la localisation des aires d'accueil dans la ville. Ainsi, un travail sur la distance entre les espaces de stationnement, les centres villes et deux services de 
proximité (gendarmerie ${ }^{2}$ et école) est initié pour discuter de la localisation géographique de ces aires d'accueil. Le constat montre inévitablement un éloignement de ces structures mais également une mise à l'écart des Voyageurs dans l'espace communal. Cependant, comme c'est le cas pour quelques aires d'accueil de la région PoitouCharentes, le phénomène de périurbanisation, constant depuis les quinze dernières années, replace l'aire d'accueil dans l'espace urbain, la transformant parfois en véritable quartier dans la ville.

Cette thèse décrypte donc en détail le quotidien et la circulation des Voyageurs. L'ensemble du matériau recueilli pendant les entretiens, les périodes d'observation et d'immersion, permet de mettre en lumière plusieurs éléments ; celui du statut d'habitant des Voyageurs - trop souvent considérés comme des personnes sans attache -; celui d'une circulation structurée et qui répond à différentes motivations inscrites dans une mémoire de la circulation; celui de l'importance familiale dans l'équilibre du voyage; enfin, celui de l'impossibilité d'établir des profils: il n'existe pas de
Voyageurs métronomes, semi-sédentaires, etc. Tous façonnent leur voyage en fonction d'habitudes de vie et de circulation, en fonction de tactiques économiques, etc. Cette thèse montre ainsi que le voyage n'est pas éteint - contrairement à la pensée commune de l'opinion publique et politique -, il s'adapte, se remodèle en fonction des obstacles qui surviennent, que ceux-ci soient politiques ou économiques.

Céline Bergeon

Docteure en géographie Migrinter - UMR 7301

CNRS /Université de Poitiers celine.bergeon@univ-poitiers.fr

\footnotetext{
${ }^{2} \mathrm{La}$ gendarmerie est un lieu essentiel dans le quotidien des familles dans la mesure où celles-ci doivent faire viser un carnet de circulation tous les trois mois.
} 